\title{
Autonomie, Motivation und Aufgabenerfüllung im Anfangsunterricht Russisch
}

\author{
Anastasia Drackert (Bochum)
}

В настоящей статье представлены результаты Авух исследований, посвященных вАиянию автономии (самоопределения) на внутреннюю мотивацию студентов и качество выполненного ими заАания. 120 студентов немецкого университета, изучающих русский язык как иностранный, работали в парах, выполняя заАание, целью которого была подготовка письменной презентации об известной мичности современной России. Свобода выбора была ограничена Авумя факторами: темой (про кого?) и содержанием (о чём?). По окончании работы над презентацией студенты Аолжны были заполнить анкету, содержащую вопросы о мотивации. Кроме того, было проанализировано качество выполненного задания на основе нескольких критериев. Исследование показало, что максимальная степень осознания автономии и мотивации и более высокое качество презентаций были зафиксированы при ограниченном наборе исходных условий в задании, что противоречит теории самодетерминации Э. Аеси и Р. Райана (1985). Результаты исследования вносят вкАаА в практическое применение теории самодетерминации на начальных стадиях обучения иностранному языку.

\section{1. $\quad$ Einleitung}

Zahlreiche Forschungsarbeiten im Bereich des Fremdsprachenlernens betreffen individuelle Unterschiede der LernerInnen (wie z. B. Alter, Geschlecht, Lernstile, Angst) und deren Einfluss auf den Lernerfolg (Dörnyei \& Ryan 2015, Pawlak 2012). Dabei wurde Motivation neben der Sprachlerneignung als einer der ,großen zwei' individuellen Faktoren erkannt (Ellis 2004: 531), sodass ihr in der

Anka Bergmann, Olga Caspers \& Wolfgang Stadler (Hg.)

Didaktik der slawischen Sprachen - Beiträge zum 1. Arbeitskreis in Berlin (12.-14.9.2016)

(C) 2018 innsbruck university press, ISBN 978-3-903187-11-5, DOI 10.15203/3187-11-5 
Fremdsprachendidaktik „die größte Erklärungskraft in Bezug auf individuelle Unterschiede zugesprochen wird“ (Riemer 2016: 267).

In der Sprachlehrforschung wurden bereits zahlreiche Motivationstheorien angewandt, u. a. das Konzept der Selbstwirksamkeitserwartung (Bandura 2001), das task-value model (Wigfield \& Eccles 2000), die Zielsetzungstheorie (Locke \& Lathham 2002), das Rubikon-Modell der Handlungsphasen (Heckhausen \& Kuhl 1985), die Akkulturationstheorie (Schumann 1986) und die possible-selves theory (Markus \& Nutrius 1986). Eine der einflussreichsten Theorien in der Motivationspsychologie ist jedoch die Selbstbestimmungstheorie von Deci \& Ryan $(1985,2000)$. Diese Theorie ist erfolgreich in die Spracherwerbsforschung eingegangen (vgl. u. a. Noels 2001, Noels, Clement \& Pelletier 1999, Noels, Clement, Pelletier \& Vallerand 2000, Tanaka 2013).

Neueste Entwicklungen im Bereich der Fremdsprachendidaktik, u. a. der Übergang zum handlungs- und kompetenzorientierten Ansatz, der in der Unterrichtspraxis durch die Arbeit mit möglichst authentischen und realitätsnahen Aufgaben realisiert wird (vgl. u. a. Müller-Hartmann \& Schocker 2016), stellen einen weiteren Grund dar, warum der Erforschung der Aufgabenmotivation eine hohe Priorität gegeben werden sollte. Eine Reihe von Untersuchungen im Bereich der Motivation von FremdsprachenlernerInnen (Dörnyei \& Kormos 2000, Dörnyei 2002, Kormos \& Dörnyei 2004) zeigen, dass LernerInnen mit demselben Grad an Eingangsmotivation für das Lernen einer bestimmten Fremdsprache, je nach Aufgabenbedingungen und Aufgabenmerkmalen unterschiedliche Grade an Aufgabenmotivation empfinden können. U. a. stellen die positive Einstellung zu einer Aufgabe sowie die Motivation des/der LernpartnersIn wichtige Faktoren für die Aufgabenmotivation dar. Da Motivation für bedeutende lernfördernde Entscheidungen verantwortlich ist, wie z. B. ob Fremdsprachenlernende an einer Aufgabe arbeiten möchten, wie lange sie bereit sind, sich einer Aufgabe zu widmen und wie intensiv sie die Aufgabe verfolgen möchten, ist es sowohl für Fremdsprachenlehrende als auch für SprachlehrforscherInnen wichtig, der Frage nachzugehen, welche Aspekte der Aufgabe auf die Lernenden motivierend wirken und als Konsequenz das Fremdsprachenlernen unterstützen.

Trotz der Bedeutung der Aufgabenmotivation wurde in der fremdsprachlichen Forschung diesem Thema bisher nur wenig Aufmerksamkeit geschenkt

Anka Bergmann, Olga Caspers \& Wolfgang Stadler (Hg.)

Didaktik der slawischen Sprachen - Beiträge zum 1. Arbeitskreis in Berlin (12.-14.9.2016)

(C) 2018 innsbruck university press, ISBN 978-3-903187-11-5, DOI 10.15203/3187-11-5 
(Ben Maad 2012, Dembovskaya 2009, Dörnyei 2002, Dörnyei \& Kormos 2000, Julkunen 1989, Kormos \& Dörnyei 2004, Yanguas 2007). Hinzu kommt, dass nur die wenigsten Forschungsarbeiten Motivation aus der Perspektive der Selbstbestimmungstheorie (Ma 2009, Mozgalina 2015) untersuchen. Diese Studie zielt deshalb darauf ab, das Thema der Aufgabenmotivation aus der Perspektive der Selbstbestimmungstheorie weiter zu erforschen und zwar im Kontext des Anfangsunterrichts des Russischen als Fremdsprache (RAF) an einer deutschen Universität.

\section{Theoretischer Hintergrund}

\subsection{Selbstbestimmungstheorie}

Die Selbstbestimmungstheorie wurde in den 1980er Jahren von den amerikanischen Psychologen Deci \& Ryan (1985) formuliert. Ihnen zufolge erleben Menschen verschiedene Arten der Motivation, die man auf einem Kontinuum darstellen kann. Am Anfang des Kontinuums befindet sich die am wenigsten selbstbestimmte Art der Motivation, die sog. Amotivation, die man erlebt, wenn man weder die erforderliche Kompetenz zur Verrichtung einer Aufgabe besitzt, noch irgendeinen positiven Wert darin sieht, eine Handlung auszuführen. Am anderen Ende des Kontinuums befindet sich die intrinsische Motivation, die empfunden wird, wenn eine Handlung aus purem Genuss oder reiner Befriedigung ausgeführt wird. Eine wiederum andere wichtige Art der Motivation stellt die extrinsische Motivation dar, die auftritt, wenn eine Tätigkeit aus einem instrumentalen Motiv vorgenommen wird. Die extrinsische Motivation ist in drei weitere Kategorien - je nach Grad der Internalisierung bestimmter Vorstellungen - unterteilt: externe Regulation, introjizierte Regulation und identifizierte Regulation. Von allen Arten der Motivation stellt die intrinsische Motivation die im höchsten Maße selbstbestimmte Art der Motivation dar, die, wie empirisch gezeigt wurde, zu größerem Engagement, höherer Vitalität und Kreativität bei

der Erfüllung von Aufgaben im Leben der Betroffenen, deren Beziehungen und Projekten führt (Deci \& Ryan 2012: 85).

Anka Bergmann, Olga Caspers \& Wolfgang Stadler (Hg.)

Didaktik der slawischen Sprachen - Beiträge zum 1. Arbeitskreis in Berlin (12.-14.9.2016)

(C) 2018 innsbruck university press, ISBN 978-3-903187-11-5, DOI 10.15203/3187-11-5 
Laut den beiden Psychologen kann intrinsische Motivation nur dann empfunden werden, wenn drei fundamentale menschliche Grundbedürfnisse befriedigt werden, nämlich Autonomie, Kompetenz und soziale Eingebundenheit. Die Befriedigung dieser drei Bedürfnisse, insb. der Autonomie, ist entscheidend für die autonome, selbstbestimmte Motivation. Soziale Kontexte, inkl. Lernkontexte, Autonomie, Kompetenz und soziale Eingebundenheit unterstützen und fördern das Erleben intrinsischer Motivation. Soziale Kontexte, die dies nicht tun, konterkarieren intrinsische Motivation.

Eine Studie von Noels, Clement \& Pelletier (1999) ist die erste Untersuchung im Bereich der Sprachlehrforschung, die Lernermotivation im Fremdsprachenunterricht aus der Perspektive der Selbstbestimmungstheorie untersuchte. Die Studie erforschte Unterrichtsstile von Fremdsprachenlehrenden und kam zu dem Ergebnis, dass FremdsprachenlernerInnen, die ihre Lehrkräfte als autonomieunterstützend wahrnahmen, ein höheres Maß an intrinsischer Motivation zeigten, als diejenigen, die ihre Lehrer als kontrollierend empfanden. Der wichtigste Beitrag von Noels et al. (2000) war die Entwicklung der Language Learning Orientation Scale, die in mehreren Studien im Bereich des Fremdsprachenlernens eingesetzt wurde.

Erst im Jahre 2009 wurde die Selbstbestimmungstheorie zum ersten Mal für das Erforschen der Aufgabenmotivation angewandt, nämlich in einer Untersuchung von Ma (2009), welche die Aufgabenmotivation koreanischer Englischlernender erforschte. Ihre Studie verdeutlichte, dass jene LernerInnen, die mehr Wahlmöglichkeiten innerhalb einer mündlichen Argumentationsaufgabe bekamen, sich zwar autonomer wahrnahmen, aber keine erhöhte Motivation aufwiesen.

Die vorliegende Untersuchung beschäftigt sich primär mit dem Bedürfnis der Autonomie und untersucht den Einfluss der in die Aufgabe eingebauten Auswahlmöglichkeiten auf die Wahrnehmung der Aufgabenbedingungen sowie die Qualität der Aufgabenerfüllung. 


\subsection{Autonomie und Wahlmöglichkeiten}

Deci \& Ryan (1985) definierten Autonomie als ungezwungene Bereitschaft, sich mit einer Aktivität zu beschäftigen. Laut ihrer Theorie können Aktivitäten oder Aufgaben mit mehr Auswahlmöglichkeiten Lernautonomie fördern, während Aufgaben mit wenigen oder keinen Auswahlmöglichkeiten diese eher hindern. Zahlreiche Studien in der Motivationspsychologie stellten einen positiven Einfluss der Autonomie (Selbstbestimmung) auf die Aufgabenmotivation, Leistung, Aufgabenerfüllung, darauffolgendes Lernen und die wahrgenommene Kompetenz in verschiedenen (Lern-)Situationen bei verschiedenen (Lern-)Populationen fest (Becker 1997, Cordova \& Lepper 1996, Deci \& Ryan 2012, Kernan, Heimann \& Hanges 1991). Diese vorhergesagten Effekte wurden auch in einer Metaanalyse von 41 Studien bekräftigt (Patall, Cooper \& Robinson 2008).

Es zu ermöglichen, selbstständig Entscheidungen zu treffen, ist die offensichtlichste Art und Weise, Autonomie zu fördern. Dabei können die Anzahl und die Arten von Entscheidungen, die ein Lerner selbst treffen kann, unterschiedlich sein. In der Auswertung einer Reihe von Studien zum Zusammenhang zwischen Autonomie und Auswahlmöglichkeiten nannte Patall (2012) mehrere Untersuchungen, in denen einer hohen Anzahl an Auswahlmöglichkeiten eher negative Konsequenzen auf Motivation und Aufgabenerfüllung zugeschrieben wurden. Dafür, ob die Auswirkungen von Wahlmöglichkeiten eher positiv oder negativ waren, spielte u. a. der Umstand eine Rolle, wie viel mehr Anstrengung und Mühe es mit sich brachte, eine zusätzliche Wahl zu treffen (Botti \& Iyengar 2006). Ein weiterer wesentlicher Faktor ist die Zahl der bereitgestellten Auswahlmöglichkeiten. Literatur aus dem Bereich der Psychologie empfiehlt eine optimale Anzahl von Optionen: Auswählen aus zwei bis vier Möglichkeiten hat positivere Konsequenzen als keine Wahl zu haben oder eine Entscheidung zwischen mehr als vier Optionen treffen zu müssen (vgl. Patall 2012).

Trotz widersprüchlicher Ergebnisse bezüglich der Auswirkung der Auswahlmöglichkeiten im Bereich der Motivationspsychologie konnten einige autonomiefördernde Merkmale von Lernaufgaben formuliert werden. Das Ziel dieser Untersuchung ist es, die Auswirkung von weiteren Aspekten der Selbstbestimmung im Kontext des Anfangsunterrichts RAF zu untersuchen.

Anka Bergmann, Olga Caspers \& Wolfgang Stadler (Hg.)

Didaktik der slawischen Sprachen - Beiträge zum 1. Arbeitskreis in Berlin (12.-14.9.2016)

(C) 2018 innsbruck university press, ISBN 978-3-903187-11-5, DOI 10.15203/3187-11-5 


\section{Forschungsfragen}

Die vorliegende Studie untersucht, ob der Autonomiegrad der Lernaufgabe einen Einfluss auf die Wahrnehmung der Aufgabe sowie auf die Qualität deren Erfüllung hat. Für die Studie wurden vier Forschungsfragen formuliert:

1. Zeigen RussischlernerInnen unterschiedliche Grade wahrgenommener Autonomie je nach Aufgabenbedingungen?

2. Zeigen RussischlernerInnen unterschiedliche Grade wahrgenommener Kompetenz je nach Aufgabenbedingungen?

3. Zeigen RussischlernerInnen unterschiedliche Grade von Aufgabenmotivation je nach Aufgabenbedingungen?

4. Unterscheidet sich die Qualität der schriftlichen Aufgabe je nach Aufgabenbedingungen?

\section{Methodisches Vorgehen}

\subsection{TeilnehmerInnen}

TeilnehmerInnen der Studie waren 120 Studierende der Ruhr-Universität Bochum, die RAF auf Niveau A1 des GeR am Zentrum für Fremdsprachenausbildung im Optionalbereich gelernt haben. Da die Studierenden sich für verschiedene Fremdsprachen im Rahmen des Optionalbereichs entscheiden konnten, kann man davon ausgehen, dass ihre Eingangsmotivation, Russisch zu lernen, relativ hoch war.

Die meisten der TeilnehmerInnen hatten Deutsch als Erstsprache ( $N=102)$, es waren aber auch einige andere Erstsprachen vertreten. Die Datenerhebung fand in der letzten Woche des auf vier Semesterwochenstunden angelegten Kurses statt und wurde in neun verschiedenen Kursen durchgeführt. Es musste jeweils eine Aufgabe unter unterschiedlichen Aufgabenbedingungen bewältigt werden, d. h. mit einem unterschiedlichen Grad der Autonomie bzw. Entscheidungsfreiheit. 


\subsection{Aufgabe(n)}

Bei der Aufgabe handelte es sich um die Vorbereitung einer Präsentation über eine berühmte Persönlichkeit Russlands aus der heutigen Zeit. Die Studierenden arbeiteten zu zweit und sollten zuerst eine Recherche im Internet über die Person durchführen. Im zweiten Schritt sollten sie eine schriftliche Version der Präsentation vorbereiten, die sie später ihren KommilitonInnen vortragen sollten. Die Präsentation sollte einem sprachlichen Niveau entsprechen, das ihre KommilitonInnen ohne Hilfsmittel verstehen konnten.

Autonomie wurde dabei hinsichtlich zweier Aspekte der Aufgabe manipuliert: Zum einen war dies der Faktor der Selbstbestimmung über die zu beschreibende Person. Der zweite manipulierte Faktor bestand in der Selbstbestimmung über die Inhalte der Präsentation. In der ersten Studie gab es drei Aufgabenbedingungen:

1. keine Wahl (KW): den Studierenden wurde eine bestimmte Persönlichkeit vorgegeben;

2. begrenzte Wahl (BW): die Studierenden konnten sich für eine der vier vorgegebenen Personen entscheiden; und

3. freie Wahl (FW): die Studierenden konnten sich eine Person aussuchen.

Dabei mussten alle drei Gruppen in Studie 1 in ihrer Präsentation zwölf vorgegebene Fragen beantworten, welche die Inhalte des A1-Kurses repräsentierten. D. h. keine der Gruppen hatte eine Wahlmöglichkeit hinsichtlich der Inhalte der Präsentation.

In Studie 2 wurden den Studierenden dieselben drei Aufgabenbedingungen gestellt, was die Wahl der Person angeht, d. h. keine Wahl, begrenzte Wahl und freie Wahl. Zusätzlich durften sich aber alle drei Gruppen in Studie 2 frei entscheiden, worüber sie die Präsentation vorbereiten, mit anderen Worten, was die Inhalte der Präsentation sein sollten. Abb. 1 veranschaulicht die sechs Aufgabenbedingungen und Tab. 1 fasst die Zuordnung der Studierenden zu den sechs Aufgabenbedingungen zusammen. 


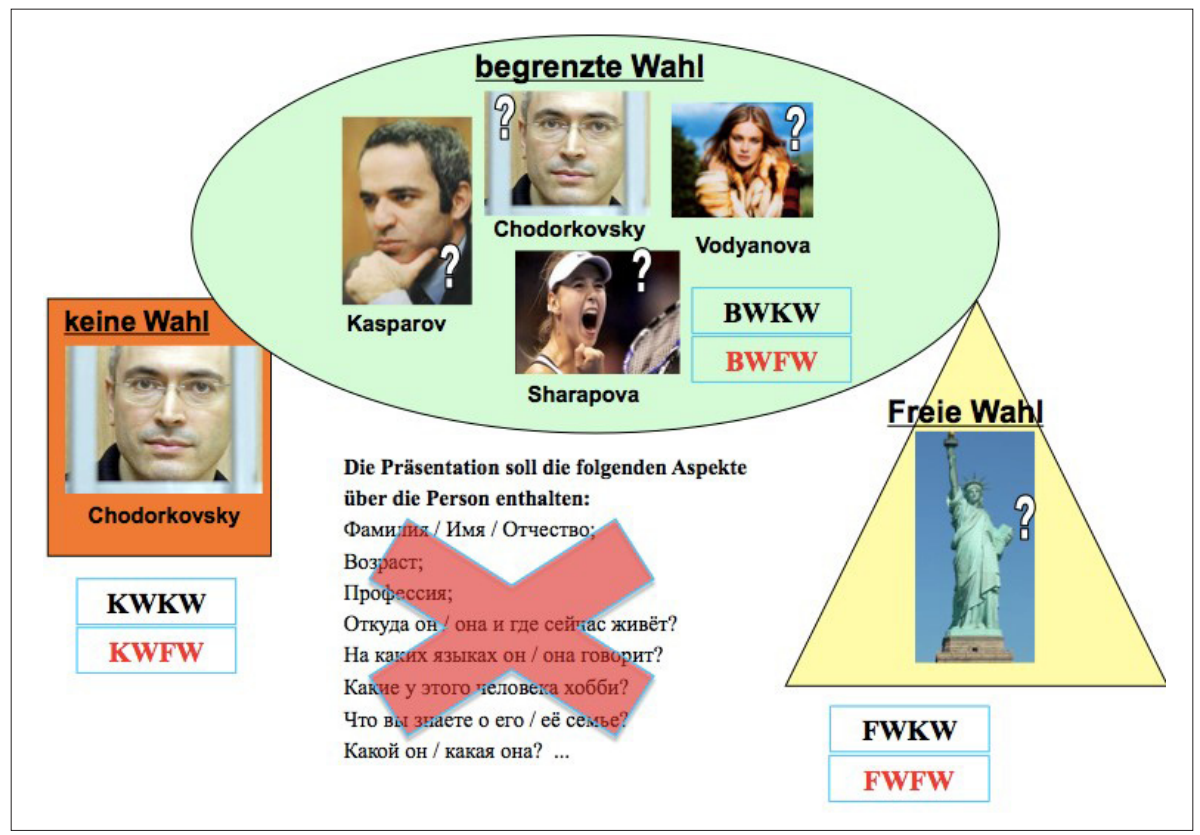

Abbildung 1: Aufgabenbedingungen

Tabelle 1: Zuordnung der Studierenden zu den sechs Aufgabenbedingungen

\begin{tabular}{|c|c|c|c|c|}
\hline Studie $(N)$ & Person & Inhalt & $\begin{array}{c}\text { Anzahl der } \\
\text { Studierenden }\end{array}$ & $\begin{array}{c}\text { Anzahl der } \\
\text { Präsentationen }\end{array}$ \\
\hline \multirow{3}{*}{$\begin{array}{c}1(N=72) \\
\text { - Autonomie beim } \\
\text { Inhalt }\end{array}$} & KW & KW & 23 & 11 \\
\hline & BW & KW & 26 & 13 \\
\hline & FW & KW & 23 & 12 \\
\hline \multirow{3}{*}{$\begin{array}{c}2(N=48) \\
+ \text { Autonomie beim } \\
\text { Inhalt }\end{array}$} & KW & FW & 14 & 7 \\
\hline & BW & FW & 21 & 10 \\
\hline & FW & FW & 13 & 6 \\
\hline
\end{tabular}




\subsection{Methode}

Nachdem die Studierenden ihre schriftliche Präsentation zu zweit vorbereitet hatten, füllten sie individuell einen Fragebogen aus, der 21 Items zu ihrer Wahrnehmung der Aufgabe in Bezug auf die drei oben beschriebenen Grundbedürfnisse (Autonomie, Kompetenz, soziale Eingebundenheit) sowie auf vier verschiedene Arten der Motivation beinhaltete. Zu jedem Konstrukt gab es jeweils drei Items, die in Form einer Likert-Skala mit sechs Punkten konstruiert wurden. Die Reliabilität des Fragebogens war im Durchschnitt $\alpha=0.76$ pro Konstrukt. Der Fragebogen findet sich in Mozgalina (2015).

Die Qualität der Aufgabenerfüllung wurde nach drei verschiedenen Kriterien beurteilt. Tab. 2 gibt einen Überblick über die Kriterien. Als erstes Kriterium der Qualität der Aufgabe dienten Bewertungen von drei Dozentinnen, die diesen A1-Kurs mind. zweimal unterrichtet hatten. Zuerst sollten sie alle Präsentationen lesen und diese im nächsten Schritt nach drei Kriterien - allgemeiner Eindruck, Inhalt und Sprache - beurteilen.

Da menschlichen Bewertungen, auch trotz eines Bewertungsrasters, ein hohes Maß an Subjektivität zugeschrieben wird, wurde entschieden, auch objektive Kriterien der Qualität der schriftlichen Präsentationen, nämlich eine Analyse der lexikalischen und syntaktischen Komplexität, durchzuführen. Diese wurden durch den Giraud's Index und die durchschnittliche Länge der T-Units ermittelt.

Zum Schluss wurden zwei alternative Kriterien für die Analyse der schriftlichen Präsentationen angewandt, nämlich die Anwendung lexiko-grammatikalischer Mittel über Niveau A1 sowie die Abdeckung der Inhalte der Präsentation. Diese Analyse wurde von zwei Dozentinnen durchgeführt, die einen A1-Kurs in Russisch mehrmals durchgeführt hatten und eine Vorstellung davon hatten, welche sprachlichen Phänomene im Unterricht vorkamen und welche nicht. Die Texte wurden nach folgenden Kriterien bewertet: 0 Punkte bekam eine Präsentation, wenn maximal zwei Wörter über Niveau A1 lagen; 1 Punkt, wenn es maximal fünf einzelne Wörter waren; 2 Punkte, wenn im Text einige Phrasen aus zwei-drei Wörtern verwendet wurden; 3 Punkte, wenn im Text Teile der Sätze über Niveau A1 lagen; und bei 4 Punkten handelte es sich um größere Abschnitte des Textes über Niveau A1.

Anka Bergmann, Olga Caspers \& Wolfgang Stadler (Hg.)

Didaktik der slawischen Sprachen - Beiträge zum 1. Arbeitskreis in Berlin (12.-14.9.2016)

(C) 2018 innsbruck university press, ISBN 978-3-903187-11-5, DOI 10.15203/3187-11-5 
Tabelle 2: Bewertungskriterien für die schriftliche Aufgabe

\begin{tabular}{|l|c|c|}
\hline \multicolumn{2}{|c|}{ Kriterien } & Form \\
\hline $\begin{array}{l}\text { Dozentinnen- } \\
\text { bewertung }\end{array}$ & $\begin{array}{c}\text { Allgemeiner Eindruck } \\
\text { Inhalt (Vielfalt der Themen und Details) } \\
\text { Sprache (Vielfalt und Akkuratheit) }\end{array}$ & $\begin{array}{c}\text { 12 Punkte / 4 pro } \\
\text { Kriterium } \\
3 \text { Bewerterinnen }\end{array}$ \\
\hline Komplexität & $\begin{array}{c}\text { Lexikalische } \\
\text { Syntaktische }\end{array}$ & $\begin{array}{c}\text { Giraud's Index } \\
\text { MLTU }\end{array}$ \\
\hline $\begin{array}{l}\text { Alternative } \\
\text { Kriterien }\end{array}$ & $\begin{array}{c}\text { Anwendung sprachlicher Mittel } \\
\text { über Niveau A1 } \\
\text { Abdeckung der Inhalte der } \\
\text { Präsentation }\end{array}$ & $\begin{array}{c}\text { 4-p. Likert-Skala } \\
2 \text { Bewerterinnen } \\
12 \text { Punkte / 1 pro } \\
\text { Inhaltspunkt }\end{array}$ \\
\hline
\end{tabular}

\section{Ergebnisse}

Forschungsfrage 1 untersuchte, ob RussischlernerInnen unterschiedliche Grade wahrgenommener Autonomie je nach Aufgabenbedingungen empfanden. Wie man Abb. 2 entnehmen kann, zeigten drei Gruppen in Studie 1 keine großen Unterschiede in der Wahrnehmung ihrer Selbstbestimmung innerhalb der Aufgabe mit einer Differenz von 0,29 Punkten zwischen den Gruppen, die keine Wahl hatten, und der Gruppe, die eine freie Wahl hatte. Allerdings konnte man große Unterschiede (2,14 Punkte) zwischen denselben Aufgabenbedingungen beobachten, wenn die Gruppen noch zusätzlich eine freie Wahl über die Inhalte der Präsentation hatten, also in Studie 2.

Laut den Ergebnissen nahm sich die Gruppe mit dem höchsten Autonomiegrad, was die tatsächlichen Auswahlmöglichkeiten angeht, als am wenigsten selbstbestimmt wahr, was den Annahmen der Selbstbestimmungstheorie widerspricht. Ein ähnliches Bild konnte man zu Forschungsfrage 2 beobachten (s. Abb. 3). Während es keine deutlichen Unterschiede zwischen den drei Gruppen in Studie 1 bezüglich der wahrgenommenen Kompetenz gab, hat die Gruppe mit dem höchsten Grad der Autonomie wieder die niedrigsten Werte gezeigt. 


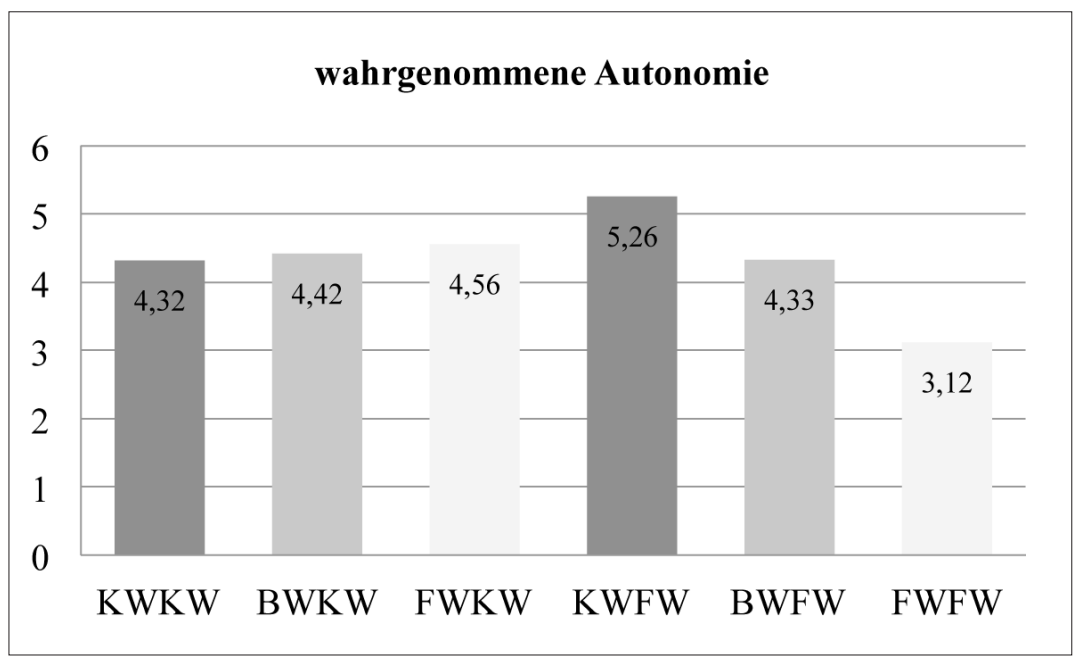

Abbildung 2: Mittelwerte für wahrgenommene Autonomie für sechs Gruppen

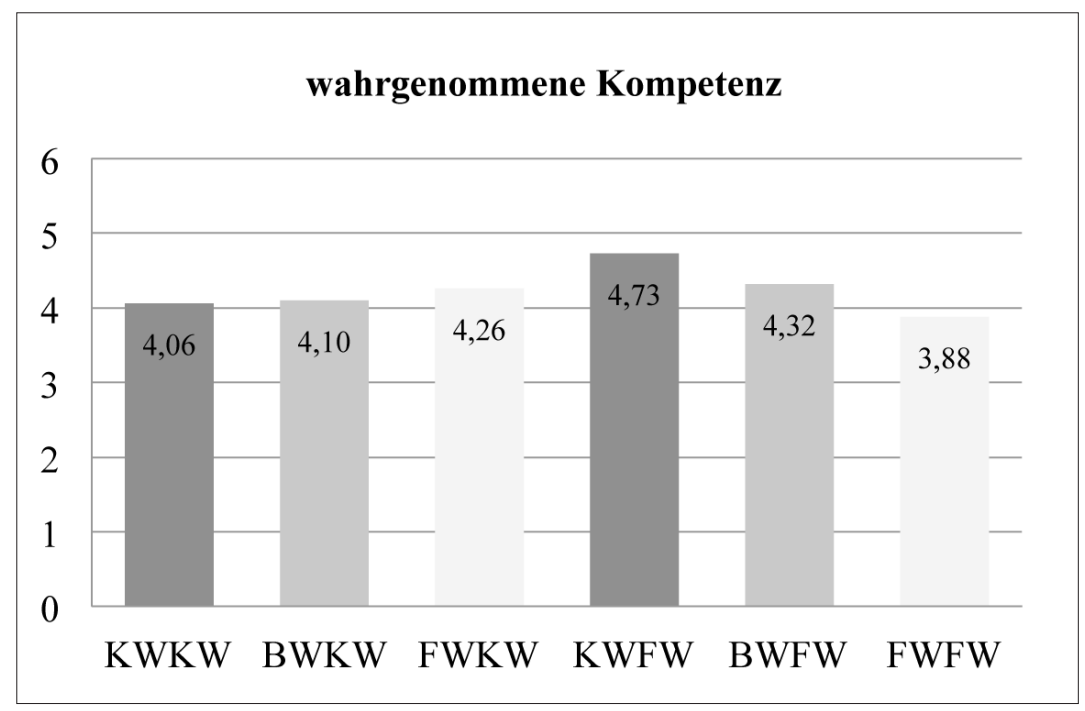

Abbildung 3: Mittelwerte für wahrgenommene Kompetenz für sechs Gruppen 
Forschungsfrage 3 untersuchte, ob die LernerInnen unterschiedliche Grade von Aufgabenmotivation je nach Aufgabenbedingungen zeigten. Es ließ sich feststellen, wie Abb. 4 zeigt, dass drei Gruppen in Studie 1 insgesamt einen hohen Grad intrinsischer Motivation und identifizierter Regulation erlebten, wie die Mittelwerte zwischen 4,06 und 4,94 zeigen. Dabei zeigten die RussischlernerInnen in der Gruppe mit begrenzter Wahl, was die zu präsentierende Person betrifft, den höchsten Grad intrinsischer Motivation $(4,39)$ und die RussischlernerInnen in der Gruppe ohne Wahlmöglichkeit den höchsten Grad identifizierter Regulation $(4,94)$. Generell kann aber nicht festgestellt werden, dass die eine oder andere Aufgabenbedingung in Bezug auf die Selbstbestimmung, was die Wahl der Person betrifft, eine Auswirkung auf die Motivation hatte.

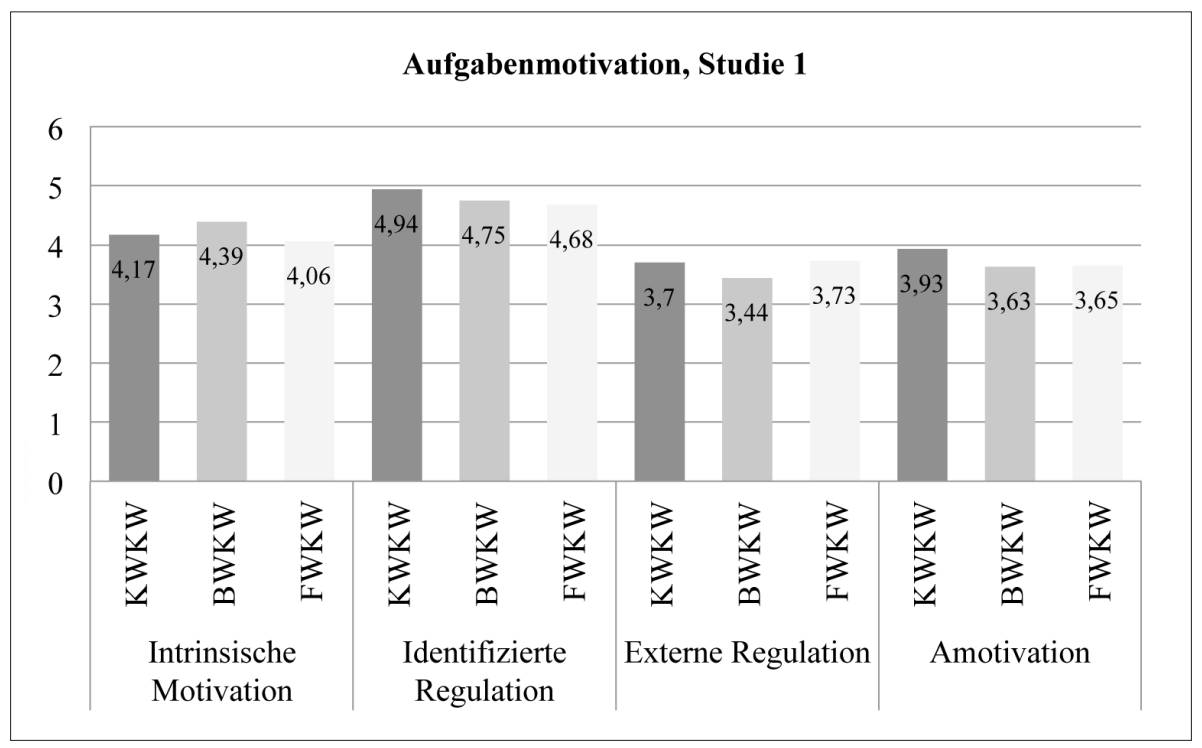

Abbildung 4: Mittelwerte für wahrgenommene Aufgabenmotivation, Studie 1

Was Studie 2 betrifft, so ließ sich feststellen, dass die Gruppe, die keine Wahl über die Person hatte, aber sich frei bezüglich der Inhalte der Präsentation entscheiden konnte, aus allen sechs Gruppen die höchsten Werte der Aufgabenmotivation aufwies. Außerdem, wie Abb. 5 zu entnehmen ist, hatten die anderen zwei Grup- 
pen in Studie 2, also die Gruppen mit der höchsten Selbstbestimmung - BWFW und FWFW -, die niedrigsten Werte.

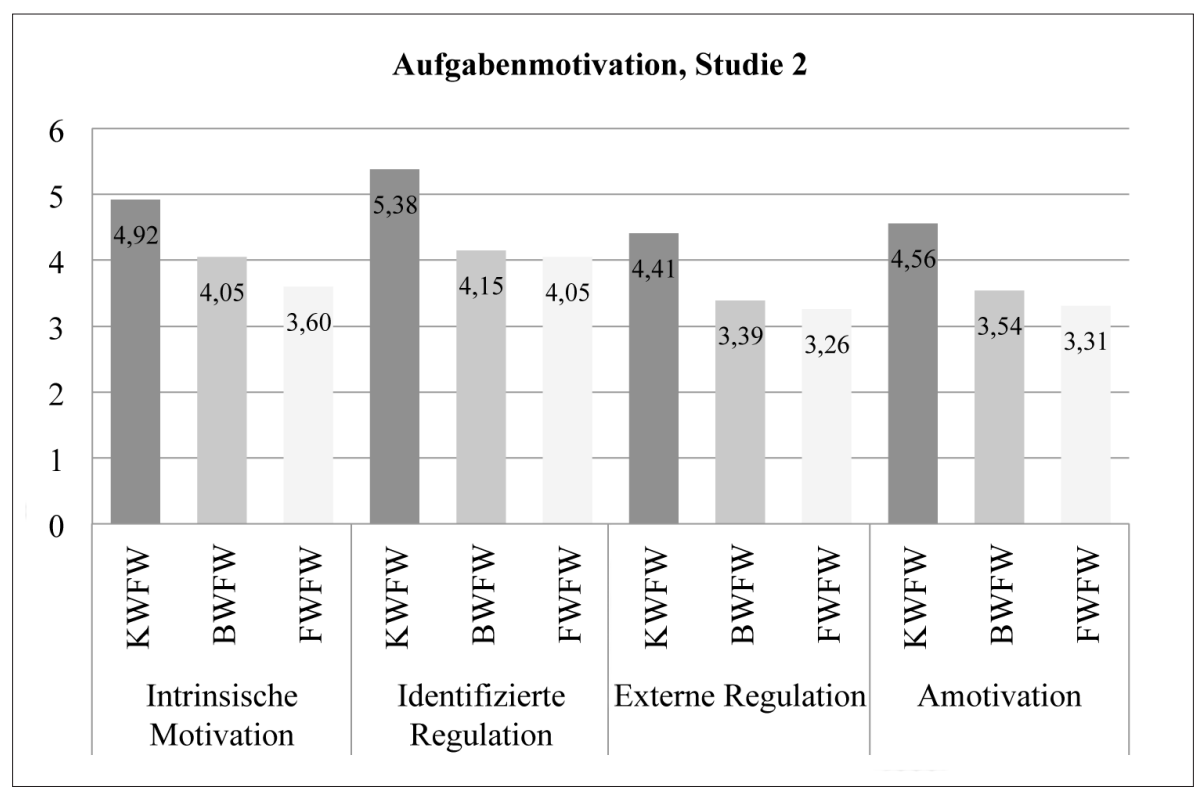

Abbildung 5: Mittelwerte für wahrgenommene Aufgabenmotivation, Studie 2

Das Ziel der Studie war es, nicht nur festzustellen, ob ein unterschiedlicher Grad an Selbstbestimmung eine Auswirkung auf die Motivation hat, sondern, ob die höhere Autonomie sich auch positiv auf die Aufgabenerfüllung auswirkt (Forschungsfrage 4). Dabei wurden der Analyse drei Kriterien zu Grunde gelegt: Dozentinnenbewertungen, lexikalische und syntaktische Komplexität und zwei alternative Methoden.

Abb. 6-8 zeigen Durchschnittsbewertungen der drei Dozentinnen für drei Kriterien und Abb. 9 präsentiert die Durchschnittswerte für die gesamte Bewertung. Wie aus den Abbildungen ersichtlich, erhielten alle Präsentationen sehr hohe Bewertungen. Dabei stellten 3 Punkte für die Vielfalt der sprachlichen Mittel und Akkuratheit den niedrigsten Wert aus vier möglichen Punkten dar. Mit dieser Punktezahl wurde allerdings Gruppe FWFW bewertet, die am meisten 
Selbstbestimmung hatte. Noch dazu, ähnlich den Ergebnissen zur Aufgabenmotivation, bekamen zwei Gruppen mit dem höchsten Grad an Selbstbestimmung, also jene Gruppen, die entweder begrenzte oder freie Wahl über die Person hatten bzw. freie Wahl, was die Inhalte der Präsentation angeht, die niedrigsten Bewertungen in allen drei Aspekten der Aufgabenerfüllung.

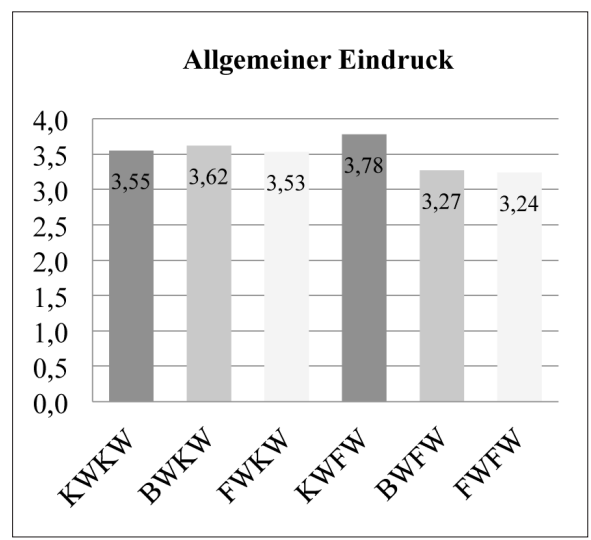

Abbildung 6: Bewertung des allgemeinen Eindrucks

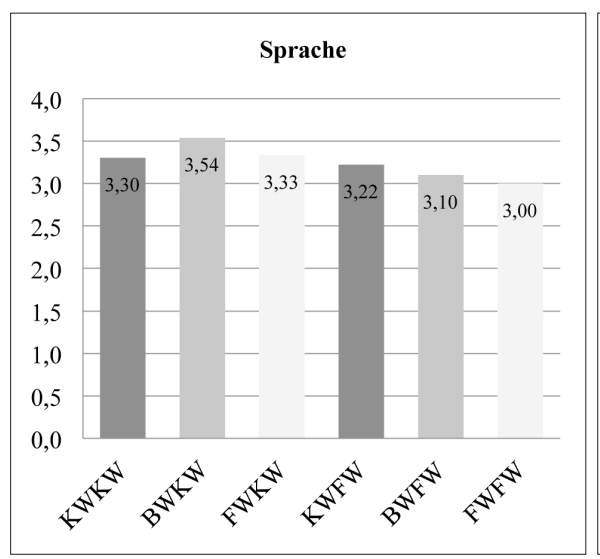

Abbildung 8: Bewertung der sprachlichen Komponente

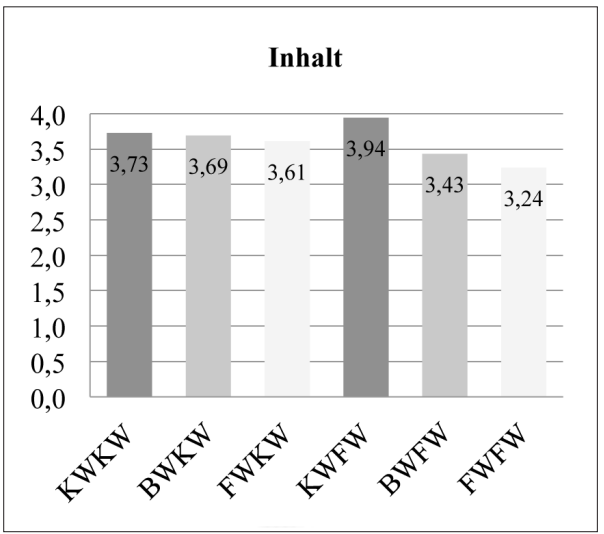

Abbildung 7: Bewertung des Inhalts

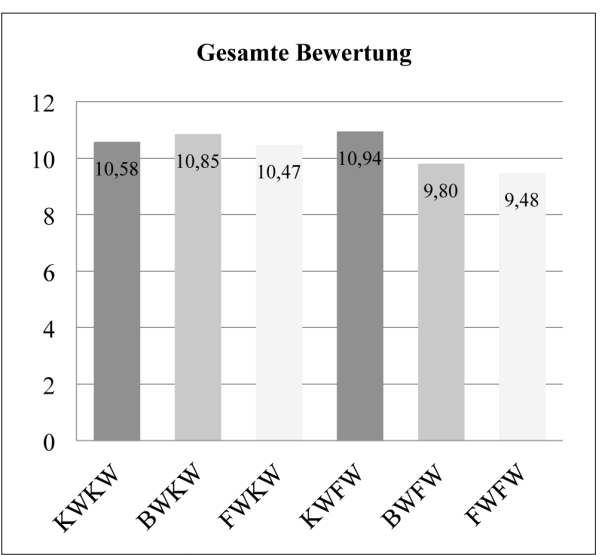

Abbildung 9: Gesamte Bewertung 
Um festzustellen, warum Russischlernende in den Gruppen BWFW und FWFW beim Bewertungskriterium Inhalt schlechter abschnitten, und um zu überprüfen, ob dies tatsächlich der Fall war, wurden die Präsentationen noch einmal in Bezug auf die Abdeckung der Inhalte analysiert. In Studie 1 bekamen die Lernenden eine Liste mit elf Fragen, die im Rahmen des Kurses behandelt worden waren, und eine weitere freie Frage. Da alle Studierenden in allen sechs Gruppen mit denselben Kursinhalten konfrontiert wurden, war davon auszugehen, dass die Studierenden in Studie 2 die vorgegebenen Inhalte in die Präsentation von sich aus einbeziehen würden. Wie Abb. 10 zeigt, deckten die Gruppen mit der höchsten Selbstbestimmung allerdings durchschnittlich nur 6,57 Fragen ab.

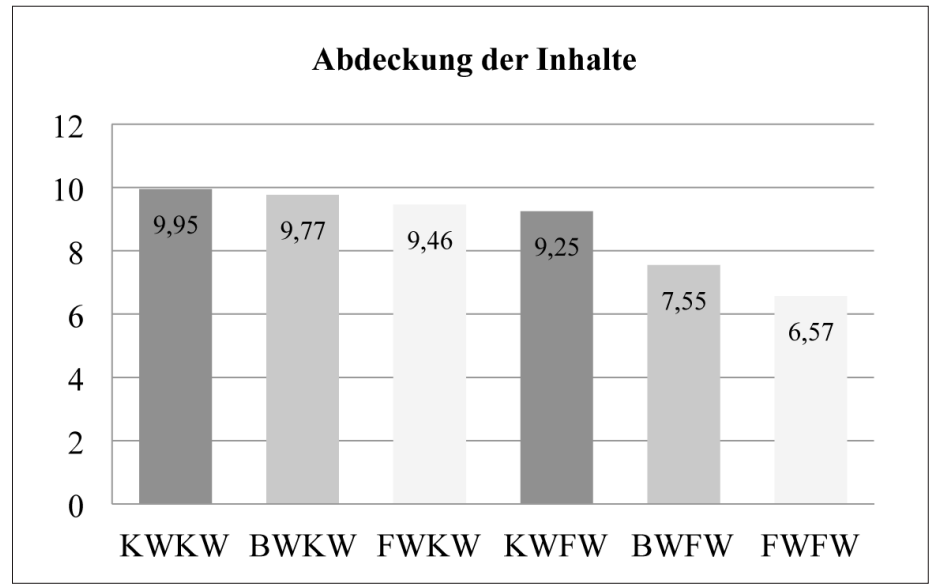

Abbildung 10: Anzahl der behandelten Fragen

Was die lexikalische und syntaktische Komplexität angeht, verdeutlichen Abb. 11 und 12 ähnliche Werte der sechs Gruppen im Bereich der lexikalischen und syntaktischen Komplexität, wobei zwei Gruppen in Studie 2 (KWFW, FWFW) ein wenig höhere Werte zeigten, was bedeutet, dass Russischlernende in diesen zwei Gruppen mehr unterschiedliche Lexeme benutzten sowie syntaktisch etwas komplexere Sätze verfassten.

Diese Ergebnisse erscheinen ein wenig widersprüchlich. Die Russischlernenden in der Gruppe mit dem höchsten Autonomiegrad (FWFW) deckten weniger 


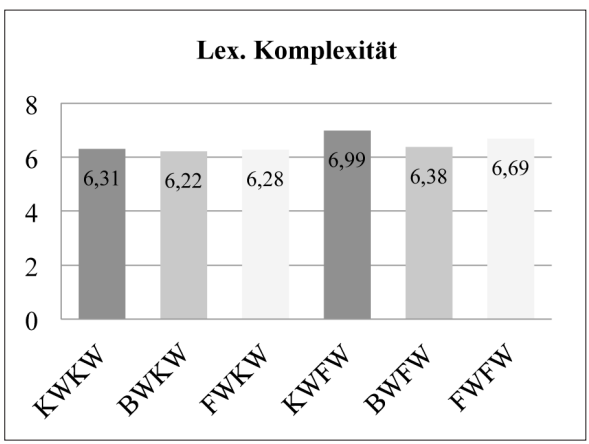

Abbildung 11: Lexikalische Komplexität

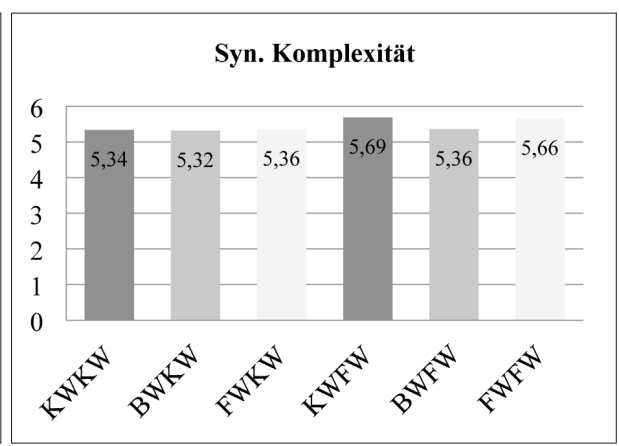

Abbildung 12: Syntaktische Komplexität

Inhalte ab, wiesen aber höhere sprachliche Komplexität auf. Außerdem merkten einige Dozentinnen bei dem Feedback zu ihren Bewertungen an, dass einige schriftliche Texte Teile enthielten, die deutlich über Niveau A1 lagen. Um festzustellen, ob es dabei bestimmte Muster in Bezug auf einzelne Gruppen gab, wurde eine weitere Analyse durchgeführt, nämlich die Analyse der Anwendung von lexiko-grammatischen Mitteln bezogen auf Niveau A1.

Abb. 13 zeigt, dass RussischlernerInnen in Studie 2 in allen drei Gruppen durchschnittlich mehr sprachliche Mittel über Niveau A1 benutzten als Russisch-

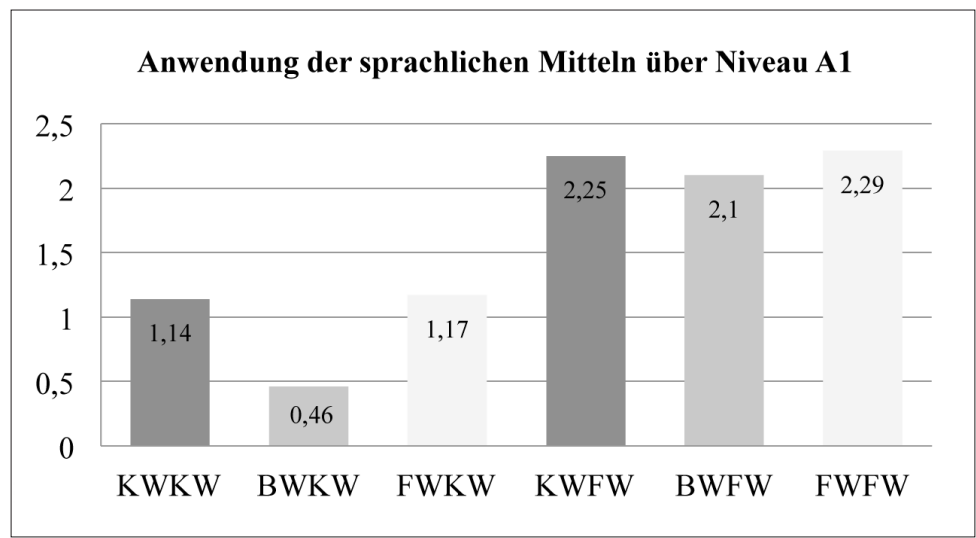

Abbildung 13: Analyse der lexiko-grammatikalischen Mittel über Niveau A1 
lernerInnen in Studie 1. Das heißt, wenn sie sich komplett frei hinsichtlich der Inhalte der Präsentation entscheiden konnten, neigten die RussischlernerInnen zur Anwendung von mehreren ihnen vorher unbekannten Wörtern und Strukturen, was für eine eindeutige Auswirkung der Selbstbestimmung auf die Textproduktion spricht.

\section{Diskussion der Ergebnisse und Ausblick}

Die vorliegende Studie beschäftigte sich mit der Frage, ob bestimmte Auswahlmöglichkeiten innerhalb einer Lernaufgabe einen Einfluss auf die wahrgenommene Autonomie und Kompetenz, die Aufgabenmotivation und die Qualität der Aufgabenerfüllung haben. Laut der Selbstbestimmungstheorie müsste dies der Fall sein: Je mehr Auswahlmöglichkeiten eine/ein Lernende/r bekommt, desto autonomer wird sie/er seine Handlungen wahrnehmen und desto höher wird ihre/seine Aufgabenmotivation sein. Als Ergebnis sollte sich des Weiteren eine höhere Qualität bei der Aufgabenerfüllung zeigen. Die Ergebnisse der Studie zeigten deutlich, dass dies im Kontext des Anfangsunterrichts RAF nicht immer der Fall ist, und zwar in Bezug auf alle untersuchten Aspekte.

\section{Wahrgenommene Autonomie}

Erstens hatte mehr Selbstbestimmung - im Gegensatz zu den Annahmen der Selbstbestimmungstheorie - innerhalb einer Lernaufgabe unterschiedliche Auswirkungen - je nachdem, wie man Autonomie definiert (s. Ergebnisse zu Forschungsfrage 1). Während in Studie 1 die Gruppen mit begrenzter oder freier Wahl sich fast gleich autonom wie die Gruppe ohne Wahlmöglichkeit einschätzte, nahmen sich Russischlernende in der Gruppe ohne Wahlmöglichkeit hinsichtlich der Person in Studie 2 viel autonomer als fünf andere Gruppen wahr, wenn sie zusätzlich frei über die Inhalte der Präsentation entscheiden konnten. Die Gruppe mit dem höchsten Grad an Selbstbestimmung empfand den

niedrigsten Grad an Selbstbestimmung aus allen sechs Gruppen. Was bedeutet das? 
Zum einen lässt sich daraus schließen, dass tatsächliche Auswahlmöglichkeiten nicht gleich als solche wahrgenommen werden. Bei der durchgeführten Aufgabe war es den Studierenden zumindest in Studie 1 anscheinend unwichtig, ob sie die Person vorgegeben bekamen, eine begrenzte oder eine freie Wahl hatten, weil sich Russischlernende in allen drei Gruppen gleich selbstbestimmt fühlten. Im Gegensatz dazu steht die Einschätzung vieler Fremdsprachenlehrender, dass mehr Auswahlmöglichkeiten ihren Lernenden mehr Freiheit ermöglichen.

Zum anderen können viele Auswahlmöglichkeiten die LernerInnen eher überfordern, als sie beim Lernen unterstützen. Einige WissenschaftlerInnen heben hervor, dass eine Auseinandersetzung mit vielen Auswahlmöglichkeiten oder ein hoher Grad an Selbstbestimmung viele kognitive Ressourcen in Anspruch nimmt (Vohs et al. 2008, Vohs, Finkenauer \& Baumeister 2010), was in Müdigkeit enden kann und in der Abnahme der Fähigkeit, selbst eine Aktivität zu initiieren und zu steuern. Dies kann besonders auf AnfängerInnen im Russischunterricht zutreffen, die überfordert sein können, wenn sie sich neben der sprachlichen Bewältigung der Aufgabe auch noch mit, übersprachlichen' Entscheidungen auseinandersetzen müssen, wie der Wahl der Person oder der Inhalte einer Präsentation. Dies befürwortet die Annahmen von Schwartz (2000: 81), der postuliert, dass unbeschränkte Freiheit zur Lähmung führt und zu einer Art selbstbesiegender Tyrannei wird.

\section{Wahrgenommene Kompetenz}

Die zweite Erkenntnis der Studie ist die Tatsache, dass die Erhöhung des Autonomiegrades einen eher negativen Einfluss auf die wahrgenommene Kompetenz haben kann. Die Studie hat gezeigt, dass mehr Freiheit zu einer schlechteren Selbsteinschätzung der RussischlernerInnen führte. Mit den durchgeführten Analysen lässt sich leider nicht eindeutig feststellen, ob die niedrigeren Werte der wahrgenommenen Kompetenz in der Gruppe mit dem höchsten Grad der Autonomie den Aufgabenbedingungen zuzuschreiben sind oder ob die 13 RussischlernerInnen in dieser Gruppe sich generell weniger kompetent einstufen. Eine Analyse der Zusammenhänge zwischen den drei Grundbedürfnissen - Autonomie, Kompetenz und soziale Gebundenheit - nicht nur als Bedingung für die Erhöhung der Motivation, sondern auch hinsichtlich ihrer Wechselwirkung,

Anka Bergmann, Olga Caspers \& Wolfgang Stadler (Hg.)

Didaktik der slawischen Sprachen - Beiträge zum 1. Arbeitskreis in Berlin (12.-14.9.2016)

(C) 2018 innsbruck university press, ISBN 978-3-903187-11-5, DOI 10.15203/3187-11-5 
kann in zukünftigen Forschungsarbeiten bessere Einblicke in die Komplexität der Zusammenhänge zwischen den drei Grundbedürfnissen und der Aufgabenautonomie ermöglichen.

\section{Aufgabenmotivation}

In Bezug auf Forschungsfrage 3, die den Einfluss der Selbstbestimmung auf die Aufgabenmotivation untersucht, konnten grundlegende Erkenntnisse gewonnen werden. Interessanterweise wirkte sich die Wahl der Person in Studie 1 kaum motivierend aus, das heißt, jene LernerInnen, die die Person vorgegeben bekamen, fanden die Aufgabe genauso motivierend wie diejenigen, die sich die Person frei aussuchen konnten. Allerdings hatte die zusätzliche Wahl der Inhalte der Präsentation deutliche Auswirkungen auf die Aufgabenmotivation - und zwar sehr positive für die Gruppe ohne Wahlmöglichkeit der Person und negative für die Gruppe mit freier Wahl der Person. Woran könnte das liegen und welche Empfehlungen ergeben sich daraus für die Praxis?

Anscheinend war die Zielaufgabe - eine Präsentation auf Basis einer Internetrecherche - motivierend genug, sodass es keine Rolle spielte, ob die LernerInnen in Studie 1 die Wahl über die Person treffen konnten oder nicht. Es wäre daher interessant zu untersuchen, ob die Auswahlmöglichkeit bei einer weniger spannenden Aufgabe oder mit einem Aufgabenformat, das sehr oft im Unterricht eingesetzt wird, eine andere Wirkung auf die Motivation hat. Die Ergebnisse der Studie 2 zeigten aber auch, dass selbst bei einer spannenden und motivierenden Aufgabe Auswahlmöglichkeiten sehr wohl wahrgenommen werden können, bspw. wenn es zu viele davon gibt. Deswegen kann möglicherweise konstatiert werden, dass eine Schwelle existiert, die definieren kann, wie viele Auswahlmöglichkeiten unterstützend wirken. Die vorliegende Studie legte deutlich dar, dass zu viele Auswahlmöglichkeiten nachteilig für die Aufgabenmotivation sein können. Es ist dementsprechend sehr wichtig, Lernaufgaben zu strukturieren, besonders in der Anfangsphase des fremdsprachlichen Unterrichts. Ob dies auch mit fortgeschrittenen LernerInnen der Fall ist, müsste zukünftige Forschung zeigen.

Ein weiterer Aspekt, der angesprochen werden soll, ist die hohe Eingangsmotivation der Russischlernenden in dieser Studie, die sicherlich einen Einfluss auf

Anka Bergmann, Olga Caspers \& Wolfgang Stadler (Hg.)

Didaktik der slawischen Sprachen - Beiträge zum 1. Arbeitskreis in Berlin (12.-14.9.2016)

(C) 2018 innsbruck university press, ISBN 978-3-903187-11-5, DOI 10.15203/3187-11-5 
die Aufgabenmotivation gehabt haben dürfte. Es wäre deshalb wichtig, weitere Studien über den Zusammenhang der Selbstbestimmung und der Aufgabenmotivation bei Lernenden durchzuführen, die von Anfang an eine eher niedrigere Motivation aufweisen, weil sie z. B. nicht freiwillig an einem Fremdsprachenunterricht teilnehmen, wie es oft an der Schule der Fall ist.

In dieser Studie wurde die Aufgabenmotivation bei schriftlichen Präsentationen analysiert. Die Analyse dieses Schritts der Aufgabenbewältigung lässt die Frage offen, ob sich die Aufgabenmotivation nach der mündlichen Präsentation ändern würde und ob die Selbstbestimmung dabei ihre Relevanz beibehält. Mit einer derartigen Analyse in einer zukünftigen Studie könnte ein besserer Eindruck über die Dynamik der Aufgabenmotivation in verschiedenen Phasen der Bewältigung einer Aufgabe gewonnen werden.

\section{Qualität der Aufgabenerfüllung}

Forschungsfrage 4 beschäftigte sich mit der Auswirkung der Motivation auf die Qualität der Aufgabenerfüllung. Es wurde festgestellt, dass die zwei Gruppen mit dem höchsten Grad der Selbstbestimmung eine etwas niedrigere Dozentinnenbewertung erhielten und weniger Themen in ihren Präsentationen behandelten, als dies aufgrund ihrer Kenntnisse zu erwarten gewesen wäre. Was allerdings die lexikalische und syntaktische Komplexität der Texte angeht, war diese tendenziell ein wenig höher als bei den Gruppen in Studie 1. Diese Erkenntnis wurde zusätzlich durch die Analyse der benutzten sprachlichen Mittel über Niveau A1 bestätigt.

Wie sind diese Ergebnisse zu bewerten? Die Antwort auf diese Frage hängt v. a. vom Ziel der Aufgabe ab. Es ist generell wünschenswert, wenn Fremdsprachenlernende von sich aus den Gebrauch neuer Lexik und Grammatik initiieren. Allerdings kann man weder Vokabeln noch Grammatik nach einmaligem Gebrauch aktiv beherrschen, was weitere Auseinandersetzung mit neuen sprachlichen Phänomenen außerhalb des Unterrichts erfordert. Wenn es bei der Aufgabe aber eher darum geht, das schon erworbene Wissen und die bereits angeeigneten Kompetenzen kreativ anzuwenden, wie es im Kontext dieser Studie der Fall war, kann zu viel Selbstbestimmung eher zu unvorhergesehenen Handlungen führen. So wurden die AnfängerInnen durch viel Selbstbestimmung eher zum Einsatz

Anka Bergmann, Olga Caspers \& Wolfgang Stadler (Hg.)

Didaktik der slawischen Sprachen - Beiträge zum 1. Arbeitskreis in Berlin (12.-14.9.2016)

(C) 2018 innsbruck university press, ISBN 978-3-903187-11-5, DOI 10.15203/3187-11-5 
von weiteren Hilfsmitteln (Wörterbüchern, Übersetzungsprogrammen etc.) angeleitet und nicht so sehr zu einer kognitiven Auseinandersetzung und kreativen Bewältigung der gestellten Aufgabe.

\section{Fazit}

Diese Studie zeigte, dass einfache Entscheidungen bei der Aufgabenkonstruktion sehr große Auswirkungen auf die Aufgabenmotivation und die Qualität der Aufgabenerfüllung haben können. Es wurde festgestellt, dass Wahlmöglichkeiten innerhalb einer Lernaufgabe eindeutig eine Auswirkung haben. Wie viele und welche Art der Auswahlmöglichkeiten sich für bestimmte Niveaustufen und bestimmte Aufgaben als motivierend und lernfördernd in einem bestimmten Lernsetting erweisen, bleibt noch zu erforschen.

\section{Literaturverzeichnis}

Bandura, A. (2001). Social Cognitive Theory: An Agentic Perspective. Annual Review of Psychology, $52,1-26$.

Becker, D. A. (1997). The Effects of Choice on Auditors' Intrinsic Motivation and Performance. Behavioral Research in Accounting, 9, 1-19.

Ben Maad, M. R. (2012). Interaction Effect of Task Demands and Individual Differences on L2 Learners' Perception of Task Difficulty and Motivation. Journal of Language Teaching and Learning, 2 (1), 1-14.

Botti, S. \& Iyengar, S. S. (2006). The Dark Size of Choice: When Choice Impairs Social Welfare. Journal of Public Policy and Marketing, 25, 24-38.

Cordova, D. \& Lepper, M. (1996). Intrinsic Motivation and the Process of Learning: Beneficial Effects of Contextualization, Personalization, and Choice. Journal of Educational Psychology, $88,715-730$.

Deci, E. L. \& Ryan, R. M. (1985). Intrinsic Motivation and Self-Determination in Human Behavior. New York: Plenum.

Deci, E. L. \& Ryan, R. M. (2000). The "What" and "Why" of Goal Pursuits: Human Needs and the Self-Determination of Behavior. Psychological Inquiry, 11, 227-268. 
Deci, E. L. \& Ryan, R. M. (2012). Motivation, Personality and Development within Embedded Social Contexts: An Overview of Self-Determination Theory. In R. M. Ryan (Hrsg.), The Oxford Handbook of Human Motivation (85-107). Oxford: Oxford University Press.

Dembovskaya, S. (2009). Task-Based Instruction: The Effect of Motivational and Cognitive Pre-Tasks on Second Language Oral French Production. Disseration, University of Iowa. Iowa.

Dörnyei, Z. (2002). The Motivational Basis of Language Learning Tasks. In P. Robinson (Hrsg.), Individual Differences and Instructed Language Learning (137-158). Amsterdam: John Benjamins.

Dörnyei, Z. \& Kormos, J. (2000). The Role of Individual and Social Variables in Oral Task Performance. Language Teaching Research, 4, 275-300.

Dörnyei. Z. \& Ryan, S. (2015). The Psychology of the Language Learner Revisited. New York: Routledge.

Ellis, R. (2004). Individual Differences in Second Language Learning. In A. Davies \& C. Elder (Hrsg.), The Handbook of Applied Linguistics (525-551). Oxford, Blackwell.

Heckhausen, H. \& Kuhl, J. (1985). From Wishes to Action: The Dead Ends and Short Cuts on the Long Way to Action. In M. Frese \& J. Sabini (Hrsg.), Goal-Directed Behaviour: The Concept of Action in Psychology (134-159). New Jersey: Lawrence Erlbaum, Hillsdale.

Julkunen K. (1989). Situation- and Task-Specific Motivation in Foreign-Language Learning and Teaching. Joensuu, Finland: University of Joensuu Publications in Education.

Kernan, M. C., Heimann, B. \& Hanges, P. J. (1991). Effects of Goal Choice, Strategy Choice, and Feedback Source on Goal Acceptance, Performance, and Subsequent Goals. Journal of Applied Social Psychology, 21, 713-733.

Kormos, J. \& Dörnyei, Z. (2004). The Interaction of Linguistic and Motivational Variables in Second Language Task Performance. Zeitschrift für Interkulturellen Fremdsprachenunterricht [Online], $9(2), 19$.

Locke, E. A. \& Latham, G. P. (2002). Building a Practically Useful Theory of Goal Setting and Task Motivation: A 35-Year Odyssey. American Psychologist, 57 (9), 705-717.

Ma, J. H. (2009). Autonomy, Competence, and Relatedness in L2 Learners' Task Motivation: A SelfDetermination Theory Perspective. Dissertation, University of Hawai'i at Manoa. Manoa.

Markus, H. \& Nutrius, P. (1986). Possible Selves. American Psychologist, 41, 954-69.

Mozgalina, A., (2015). More Choice or Less Choice? The Influence of Autonomy on Task Motivation and Task Engagement. System, 49, 120-132.

Anka Bergmann, Olga Caspers \& Wolfgang Stadler (Hg.)

Didaktik der slawischen Sprachen - Beiträge zum 1. Arbeitskreis in Berlin (12.-14.9.2016)

(C) 2018 innsbruck university press, ISBN 978-3-903187-11-5, DOI 10.15203/3187-11-5 
Müller-Hartmann, A. \& Schocker, M. (2016). Aufgabenorientierung. In. K.-R. Bausch, E. Burwitz-Melzer, H.-J. Krumm, G. Melhorn \& C. Riemer (Hrsg.), Handbuch Fremdsprachenunterricht (6., vollst. neu bearbeit. Aufl.) (325-329). Tübingen: Francke.

Noels, K. A. (2001). Learning Spanish As a Second Language: Learners' Orientations and Perceptions of Their Teachers' Communication Style. Language Learning, 51, 107-144.

Noels, K. A., Clement, R. \& Pelletier, L. G. (1999). Perceptions of Teachers' Communicative Style and Students' Intrinsic and Extrinsic Motivation. The Modern Language Journal, 83, 23-34.

Noels, K. A., Pelletier, L. G., Clement, R. \& Vallerand, R. J. (2000). Why Are You Learning a Second Language? Motivational Orientations and Self-Determination Theory. Language Learning, 50, 57-85.

Patall, E. A., Cooper, H. \& Robinson, J. C. (2008). The Effects of Choice on Intrinsic Motivation and Related Outcomes: A Meta-Analysis of Research Findings. Psychological Bulletin March 2008, 134 (2), 270-300.

Patall, E. A. (2012). The Motivational Complexity of Choosing: A Review of Theory and Research. In R. M. Ryan (Hrsg.), The Oxford Handbook of Human Motivation (248-279). Oxford: Oxford University Press.

Pawlak, M. (Hrsg.) (2012). New Perspectives on Individual Differences in Language Learning and Teaching. Berlin: Springer.

Riemer, C. (2016). Affektive Faktoren. In K.-R. Bausch, E. Burwitz-Melzer, H.-J. Krumm, G. Melhorn \& C. Riemer (Hrsg.), Handbuch Fremdsprachenunterricht. (6., vollst. neu bearbeit. Aufl.) (266-270). Tübingen: Francke.

Ryan, R. M. (Hrsg.) (2012). The Oxford Handbook of Human Motivation. Oxford: Oxford University Press.

Schumann, J. H. (1986). Research on the Acculturation Model for Second Language Acquisition. Journal of Multilingual and Multicultural Development, 7, 379-392.

Schwartz, B. (2000). The Tyranny of Freedom. American Psychologist, 55 (1), 79-88.

Tanaka, M. (2013). Examining Kanji Learning Motivation Using Self-Determination Theory. System, 41 (3), 804-816.

Vohs, K. D., Finkenauer, C. \& Baumeister, R. F. (2010). The Sum of Friends' and Lovers' SelfControl Scores Predicts Relationship Quality. Social Psychological and Personality Science, 2 (2), $138-145$.

Anka Bergmann, Olga Caspers \& Wolfgang Stadler (Hg.)

Didaktik der slawischen Sprachen - Beiträge zum 1. Arbeitskreis in Berlin (12.-14.9.2016)

(C) 2018 innsbruck university press, ISBN 978-3-903187-11-5, DOI 10.15203/3187-11-5 
Vohs, K. D., Baumeister, R. F., Schmeichel, B. J., Twenge, J. M., Nelson, N. M. \& Tice, D. M. (2008). Making Choices Impairs Subsequent Self-Control: A Limited-Resource Account of Decision Making, Self-Regulation, and Active Initiative. Journal of Personality and Social Psychology, 94 (5), 883-898.

Wigfield, A. \& Eccles, J. (2000). Expectancy-Value Theory of Achievement Motivation. Contemporary Educational Psychology, 25, 68-81.

Yanguas, Í. A. (2007). A Look at Second Language Learners' Task Motivation. Anuario del Seminario de Filología Vasca Julio de Urquijo, 41 (2), 333-345. 